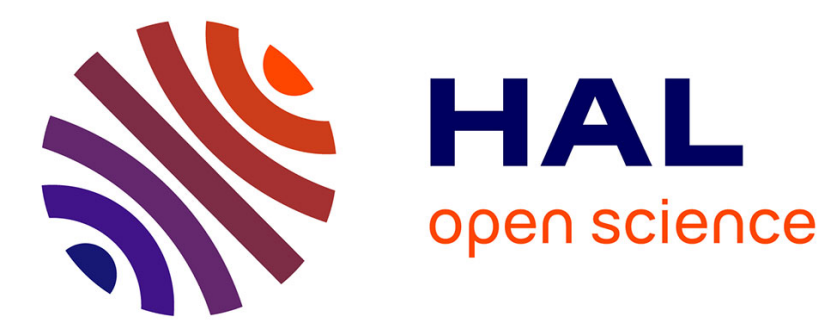

\title{
Performance analysis of compaction techniques for map representation in geographic databases
}

\author{
P. Boursier, M. Scholl
}

\section{To cite this version:}

P. Boursier, M. Scholl. Performance analysis of compaction techniques for map representation in geographic databases. RR-0058, INRIA. 1981. inria-00076503

\section{HAL Id: inria-00076503 \\ https://hal.inria.fr/inria-00076503}

Submitted on 24 May 2006

HAL is a multi-disciplinary open access archive for the deposit and dissemination of scientific research documents, whether they are published or not. The documents may come from teaching and research institutions in France or abroad, or from public or private research centers.
L'archive ouverte pluridisciplinaire HAL, est destinée au dépôt et à la diffusion de documents scientifiques de niveau recherche, publiés ou non, émanant des établissements d'enseignement et de recherche français ou étrangers, des laboratoires publics ou privés. 


\section{PERFORMANCE ANALYSIS OF COMPACTION TECHNIQUES FOR MAP REPRESENTATION IN GEOGRAPHIC DATABASES}

Instur.t.

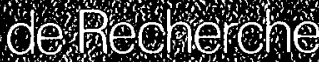
entofor

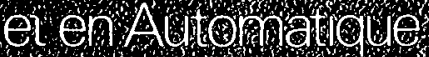

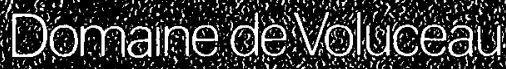
Rocotereour BP10 -653 le Chesnay Ceolex France: Тî́: 954902

Patrice BOURSIER Michel SCHOLL 


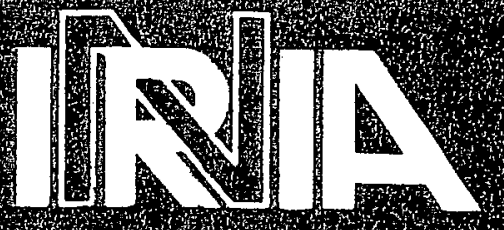

ก 40. บenn 2. 1) A A

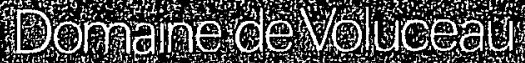

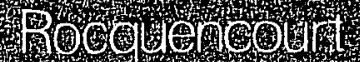
-5

2. Era.t. 1.6.

\section{Rapports de Recherche}

$$
\mathrm{N}^{\circ} 58
$$

\section{PERFORMANCE ANALYSIS OF COMPACTION TECHNIQUES FOR MAP REPRESENTATION IN GEOGRAPHIC DATABASES}

Patrice BOURSIER Michel SCHOLL

Mars 1981 


\section{PERFORMANCE ANALYSIS OF COMPACTION TECHNIQUES \\ FOR MAP REPRESENTATION IN GEOGRAPHIC DATABASES}

$=:=:=:=:=:=:=$

Patrice BOURSIER* Miche1 SCHOLL**

\section{ABSTRACT :}

A new compaction scheme for representing cellular thematic maps in 'geographic databases is presented. The memory saving provided by this method, called Hierarchical Compaction Scheme (HCS) is analyzed and compared to that of two more classical cellular schemes. HCS not only provides a significant compaction ratio but also renders processing operations such as map intersection or union easier. It also allows the use of variable scale.

\section{RESUME :}

Une nouvelle méthode de représentation compacte des cartes géographiques en mémoire est présentée. Le gain en espace mémoire que procure cette méthode cellulaire appelée Compactage Hiérarchique $(\mathrm{CH})$ est analysé et comparé au gain obteriu avec deux méthodes celluilaires plus classiques. Non seulement $\mathrm{CH}$ procure un gain en espace mémoire important, mais encore $i l$ rend les opérations ensemblistes sur les cartes, telles que l'intersection ou l'union plus faciles. 11 permet également l'utilisation d'échelles variables.

* ORSTOM, 93140 Bondy, France

${ }^{* *}$ INRIA, 78153 Le Chesnay Cedex, France 
The existing techniques for representing and processing map data in geographic databases may be partitionned into two categories as defined in [13] :

- in the polygonal techniques $[6,9,11]$ any object in the map (corn field or town, for example) is represented by its contour. Coding schemes are used for storing the contour lines.

- on the contrary, in the cellular techniques [1,3] a gridding is first applied, then all the cells "belonging" to the object are stored. The scheme compactness becomes therefore of paramount importance, especially if the purpose of encoding is primarily storage or transmission of data. However, the storage efficiency resulting from a good compaction scheme may be outweighted by an increased complexity in processing, encoding and decoding the data.

The techniques studied in this paper fall into the second group. A thematic map is used for representing a geographic attribute (such as 1 and use or slope) that may be divided into elementary values or classes of values to each of which corresponds an elementary map [3]. Each of these elementary maps is coded by means of a binary matrix that may be processed as a binary string. There are then as many binary strings as there are attribute values and it is necessary to reduce this considerable amount of storage that would otherwise prohibit the use of such methods.

The Hierarchical Compaction Scheme (HCS), introduced in [4], fulfills this compactness objective. This technique, derived from a well-known binary string. compaction technique [8], allows the database designer to represent a map under various scales. Besides, the set operations on maps are easily processed with such a data structure (see Section 2).

HCS is compared with respect to storage efficiency to two-more classical compaction techniques. The first one is a variant of Run-Length Coding (RLC) [10] while the second one called Mixed Compaction Scheme (MCS) has been implemented in [3]. An analysis of compaction ratio under HCS, RLC and MCS is reported in [4] which is based on very restrictive assumptions. We give below a new analysis of compaction ratio under RCL (Section 3) and MCS (Section 4) based on less restrictive assumptions (HCS could not be analyzed under the latter assumptions). 
In order to test the validity of the analysis, memory saving under these three techniques has been estimated from a sample of data actually stored in a geographical information system built for the Emilia-Romagna region [2,3] (Section 5).

The analytical prediction being very pessimistic, a simulation was run which leads to a more realistic prediction of the compaction ratio (Section 6 ).

In the sequel of the paper, we assume the data to be compacted is a $\mathrm{N}$ bits long string and the expected memory saving (compaction ratio) $G(N)$ is given by: $G(N)=1-S(N) / N$, where $S(N)$ is the expected length of the string after compaction.

\section{2 - HIERARCHICAL COMPACTION SCHEME (HCS)}

We describe below two variants of this scheme respectively denoted by $\mathrm{HC}_{0}$ and $\mathrm{HC}_{1}$.

\section{1 - Description}

The coding scheme is derived from the following block-compaction technique $[7,8]$ : the initial string $C_{0}$ is cut into blocks of equal length $u$ bits.

Without loss of generality, assume $N=u^{\ell}$. After compaction, $C_{0}$ is represented by a string $C_{1}$ composed of a "data string" $J_{1}$ of smaller size preceded by an index $I_{1}$. The index is organized as follows : there is a bijection between the $\frac{N}{u}$ blocks of the initial string $C_{0}$ and the $\frac{N}{U}$ bits of the index. Each index bit is set to zero if the corresponding block in $C_{0}$ is full of zeros. Such a block is called a 0-block. Otherwise, the index bit is set to one. The data string $J_{1}$ is obtained by suppressing in the initial string a 11 the 0-blocks. Figure la and $1 \mathrm{~b}$ display an example of string and its representation after compaction.

We generalize this scheme by compacting in turn the index $I_{1}$ using the same above technique. $I_{1}$ is compacted into an index $I_{2}$ and a data string $J_{2}$. Therefore, after two compactions, the initial string $C_{0}$ has been transformed into a string $\mathrm{C}_{2}$ composed of an index $\mathrm{I}_{2}$, followed by a data string $\mathrm{J}_{2}$, followed by the data string $J_{1} . I_{2}$ may be in turn compacted, etc...

Figure $1 c$ shows the representation of string $C_{0}$ after two compactions. 
(a)

$c_{0}$

\begin{tabular}{|l|l|l|l|l|l|l|l|}
\hline 0000 & 0000 & 0000 & 0000 & 1111 & 1000 & 0011 & 0000 \\
\hline
\end{tabular}

(b) $\mathrm{C}_{1}$

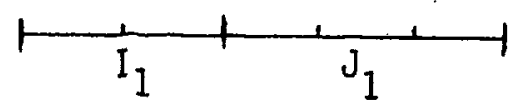

\begin{tabular}{|l|l|l|l|l|}
\hline 0000 & 1110 & 1111 & 1000 & 0011 \\
\hline
\end{tabular}

(c) $\mathrm{C}_{2}$

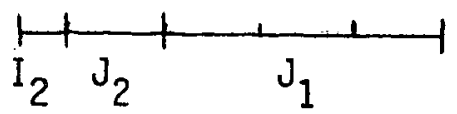

\begin{tabular}{|l|l|l|l|l|l|}
\hline 01 & 1110 & 1111 & 1000 & 0011 \\
\hline
\end{tabular}

Figure_1 : HCSS, variant HCO $_{2}, \underline{u}=\mathbf{E}=4$

As a matter of fact, for a given string of size $N$, there is an optimal

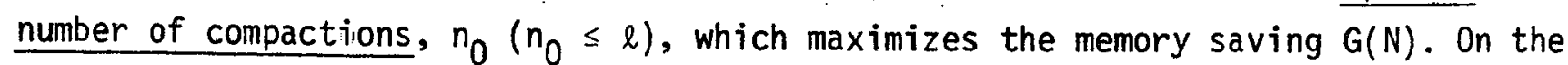
example of Figure $1, n_{0}=2$.

The memory saving achieved by this method is obviously better when the proportion of "0-bits" in the string, $p$, is high (greater or equal to 90 per cent). Conversely, when $p$ is very low, the 1-bits have to be compacted in the place of the 0-bits.

In constrast with the above method $\mathrm{Called} \mathrm{HCO}$, in the variant called $\mathrm{HCl}$, both 0-blocks and 1-blocks are compacted. At each compaction level, a "data-block" (of size $u$ bits) is represented in the index by 2 bits, ass follows : the codes 00 , 11 and 01 are respectively assigned to a 0-block, a 1-block and any other block.

Figure 2 illustrates variant $\mathrm{HCl}$ on the example of Figure 1.
(a) $C_{0}$
\begin{tabular}{|l|l|l|l|l|l|l|l|}
\hline 0000 & 0000 & 0000 & 0000 & 1111 & 1000 & 0011 & 0000 \\
\hline
\end{tabular}
(b) $\mathrm{C}_{1}$

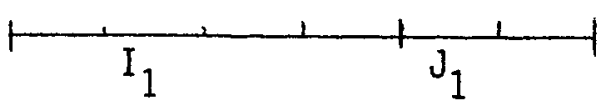
\begin{tabular}{|l|l|l|l|l|l|}
\hline 0000 & 0000 & 1101 & 0100 & 1000 & 0011 \\
\hline
\end{tabular}
(c) $\mathrm{C}_{2}$

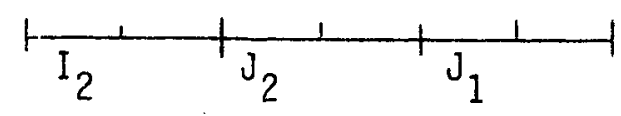
\begin{tabular}{|l|l|l|l|l|l|}
\hline 0000 & 0101 & 1101 & 0100 & 1000 & 0011 \\
\hline
\end{tabular}
(d) $\mathrm{C}_{3}$

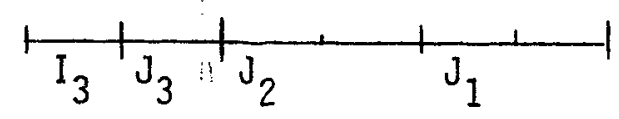
\begin{tabular}{|l|l|l|l|l|l|}
\hline 0001 & 0101 & $1101 / 0100$ & $1000 / 0011$ \\
\hline
\end{tabular}
(e) $\mathrm{C}_{4}$

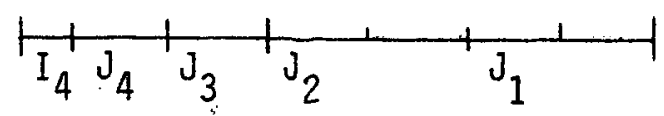

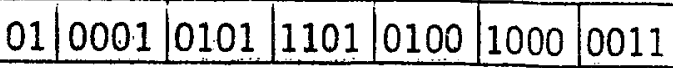

Figure_2 : $\mathrm{HCS}_{2}$ variant $\mathrm{HC} 1_{2} \underline{u}_{2}=4$ 
The optimal compaction level is now $n_{0}=1$ with a size of 24 bits for $c_{1}$. Another variant of HCS may be found in [5].

\section{2 - Anatysis}

We give in this section, the expected memory saving $G(N)$ as defined in Eq. (1) under the following assumption :

Al : Let $X_{i}$ be a random variable representing the value of the $i$ th bit in the string. We assume the $X_{i}{ }^{\prime} s, i \in[1, N]$ are independent and identically distributed : $P\left\{X_{i}=0\right\} \triangleq p$.

Consequently, the probability for any block to be a 0-block (resp. 1-block) is equal to $p^{u}$ (resp. $\left.(1-p)^{u}\right)$.

The validity of this assumption will be tested on a sample of actual data in Section 5 .

It is shown in [4] that the memory saving achieved by $\mathrm{HCO}$ is equal to :

(2) $\quad G(N)=\sum_{i=1}^{n_{0}} \frac{u^{u^{i}}-1}{u^{i}}$

where $n_{0}$ is such that:

(3) $u p^{u^{n_{0}}}>1$ and $u p^{u^{n_{0}+1}} \leq 1$

while for $\mathrm{HCl}$, we have :

$$
G(N)=\sum_{i=1}^{n_{0}} \frac{u / 2\left[p^{u^{i} / 2^{i-1}}+(1-p)^{u^{i} / 2^{i-1}}\right]-1}{u^{i} / 2^{i}}
$$

where $n_{0}$ is such that :

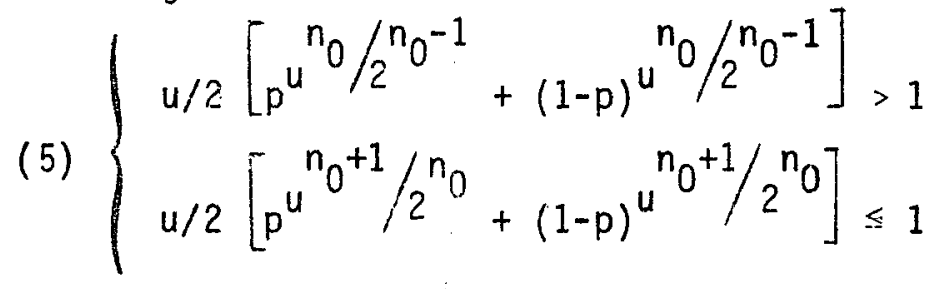




\section{3 - Discussion}

$G(N)$ is plotted versus $u$ (Eqs (2) and (4)) for both HCO (plain curves) and $\mathrm{HCl}$ (dotted curves), and for various values of $\mathrm{p}$ in Figure 3. The curves are labelled by the corresponding values of $n_{0}$.

First, note that a significant memory saving is obtained with these compaction schemes. Indeed, if $p=.999$, about 99 per cent of the memory is saved and if $p=.99$, we need only $\frac{N}{10}$ bits for storing a string of $N$ bits $(G(N) \simeq .90$ with $H C O)$. However $G(N)$ decreases fast as $p$ decreases. Intuitively, even for values of $p$ around .95 , one should expect a saving larger than that predicted by our model. In Section 5 , we show that indeed the model is pessimistic when $p$ is not close to 1 .

Second, observe that the larger $u$, the smaller the memory saving. Then, on the one hand, $u$ should not be too large (the larger $u$, the smaller the probability of a block being full of zeros, then the lower $G(N)$ ). But on the other hand, if $u$ is too small, the saving may be outweighted by the index overtiead (increasing as $u$ decreases!. Thus an optimal value of $u$ exists which maximizes $\mathrm{G}(\mathrm{N})$.

Third, it is worth nothing that HCO always provides a better performance than HCl does, i.e. the saving due to the 1-blocks compaction does not outweigh the index extraoverhead with $\mathrm{HCl}$ (two bits are needed for coding the index with $\mathrm{HCl}$ while only one bit is necessary with $\mathrm{HCO}$ ).

\section{3 - RUN-LENGTH COOING (RLC)}

This compaction technique is derived from the well-known "run-length coding" technique described in [10], when only two values may appear in the initial string, 0 and 1 .

\section{1 - Description}

Any string to be compacted being a sequence starting with a run of zeros (ones) followed by a run of ones (zeros) in turn followed by a run of zeros (ones), etc.... the RLC technique consists in representing the sequence of runs by their respective lengths, each length being coded with one block of u bits. 


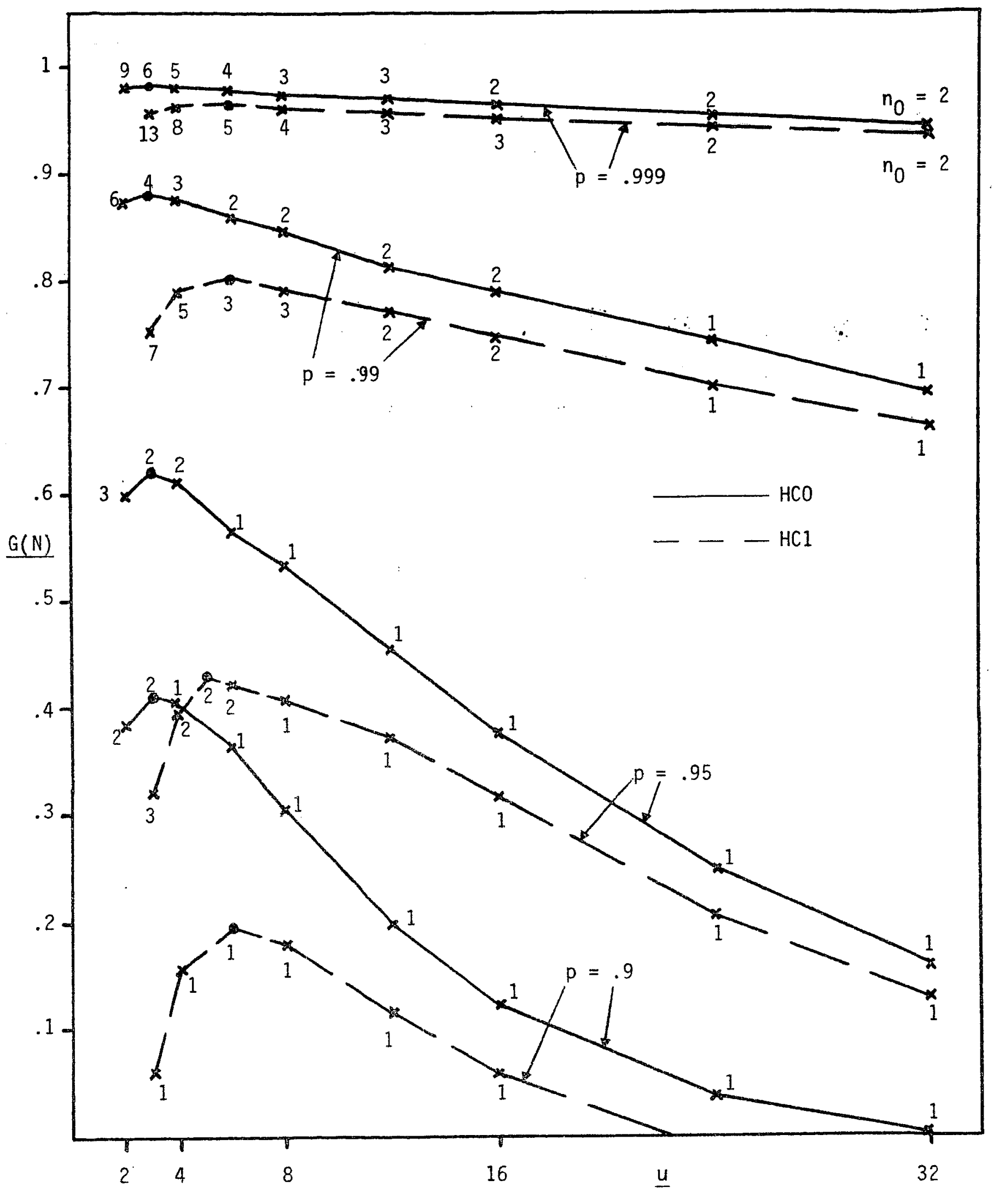

Figure 3 : HCS : memory saving versus block size u for various values of p. 
In the case where the length is larger than $\left(2^{\mathrm{u}}-1\right)$ bits, more than one block is necessary. More general1y, if the length is such that $i\left(2^{u}-1\right)<\ell \leq(i+1)\left(2^{u}-1\right)$, then we need $(i+1)$ blocks where the $i$ first blocks are full of 1 . Figure 4 shows examples of run-lengths with their respective representations.

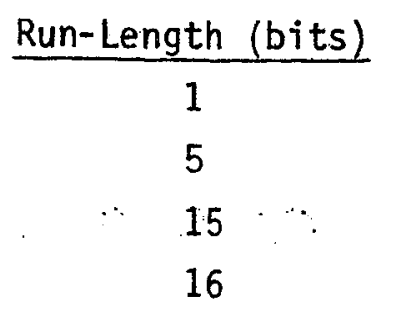

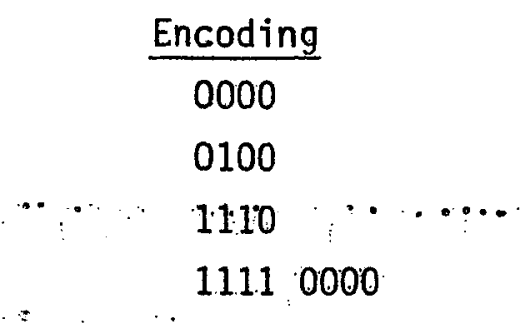

Figure 4 : Run-Length_encoding,_u_=.4

Figure 5 shows how the string $C_{0}$ of Figure la is represented after a run-length encoding has been applied $(u=4)$. Note an additional bit is necessary to indicate whether the string starts with a 0 -run or a 1-run.

$C_{4}: 0111100000100010000010011$

Figure_. 5 : Run-length_coding, ${ }_{\text {u__ }}=\mathbf{4}$

\section{2 - Analysis}

Assume without loss of generality the initial string $C_{0}$ starts with a o-run. $C_{0}$ may be considered as a sequence of subchains, each of them including a 0 -run followed by a 1-run.

Let us also assume :

A2 : the lengths of the subchains are independent and identically distributed according to any distribution function.

The subchains sequence may then be considered as a reward renewal process [14] where the occurrence of the first D-bit of a subchain is a renewal point and the associated reward is the representation "cost" of this subchain in number of blocks of size u bits. We therefore have:

(6) $\quad \lim _{N \rightarrow \infty} \frac{S(N)}{N}=\frac{E(Y)}{E(X)}$ 
where $E(X)$ is the average length of a (non-compacted) subchain and $E(Y)$ the average "cost" of the subchain compacted representation, i.e. the average number of bits necessary for the compacted representation.

Eq. (6) is true for any length distribution (as long as assumption A2 holds). In appendix $A, E(X)$ and $E(Y)$ are derived for a particular case corresponding to the following assumption (less restrictive than assumption $A 1$ of Section 2) :

Recall $x_{i}$ is a random variable representing the value of the ith bit in the string. Then assume $x_{i}$ is a Markov chain of order 1 ; i.e. more specifically, we have:

$$
\text { A3 }\left\{\begin{array}{l}
P\left\{X_{i+1}=0 \mid X_{i}=0\right\} \triangleq P_{0} \\
P\left\{X_{i+1}=1 \mid X_{i}=1\right\} \triangleq q_{1}
\end{array}\right.
$$

Then the expected length of a 0 -run is $p \bar{l}=1 / 1-p_{0}$, while that of a 1 -run is $(1-p) \bar{l}=1 / 1-q_{1}$, where $p$ is the "proportion" of 0-bits in the string and $\bar{l}$ is the expected length of a subchain.

Then we have for $N$ large (see Appendix A) :

(7) $\quad E(X)=\left(2-p_{0}-q_{1}\right) /\left[\left(1-p_{0}\right)\left(1-q_{1}\right)\right]$

(8) $\quad E(Y)=u \sum_{j=0}^{j} \max ^{-1}\left[p_{0}^{j\left(2^{u}-1\right)}+q_{1}^{j\left(2^{u}-1\right)}\right]$, where $j_{\max }=\left\lceil N /\left(2^{u}-1\right)\right\rceil$

Combining Eqs (6), (7) and ( $(3)$, we have :

(9) $\lim _{N \rightarrow \infty} \frac{S(N)}{N}=\frac{\left(1-p_{0}\right)\left(1-q_{1}\right)}{2-p_{0}-q_{1}} u \sum_{j=0}^{j_{\max ^{-1}}}\left[p_{0}^{j\left(2^{u}-1\right)}+q_{1}^{j\left(2^{u}-1\right)}\right]$

The term in the sum rapidiy becomes negligible as $j$ increases. Consequentiy, the right-hand side of Eq. (9) is a good approximation of $S(N) / N$ as $N$ is large.

The memory saving is then :

$$
G(N) \simeq 1-\frac{\left(1-p_{0}\right)\left(1-q_{1}\right)}{2-p_{0}-q_{1}} u \sum_{j=0}^{j} \max ^{-1}\left[p_{0}^{j\left(2^{u}-1\right)}+q_{1}^{j\left(2^{u}-1\right)}\right]
$$




\section{3 - Discussion}

$G(N)$ is plotted (Eq. (10)) versus $u$ in Figure 6 for $\bar{l}=100$ and for various values of $p$. As for HCS, there is an optimal block size : indeed, if $u$ is chosen too sma11, many blocks may be necessary to code a run-length. If $u$ is chosen too large, space is lost into the blocks for coding lengths which, most of the time, are much smaller than $\left(2^{u}-1\right)$.

Note that the memory saving is more sensitive to variations in the block size $u$ than with HCS. In Figure $7, G(N)$ is plotted versus (1-p) for $u=8$ and for various values of $\bar{l}$ : Observe that $G(N)$ is not sensitive to a variation in $p$. Indeed, a subchain is always represented by two blocks (one for the 0-run and one for the 1-run), although very seldom we need more than 1 block for representing a 0 -run, (when $p$ is very close to 1 ). As expected, $G(N)$ increases as $\bar{l}$ increases : obviously, since a subchain is always represented by two blocks, the larger the subchain, the larger $G(N)$.

\section{4 - MIXED COMPACTION SCHEME (MCS)}

\section{1 - Description}

The scheme studied in this section is very similar to that used in the geographic database described in [3]. The initial string $C_{0}$ is cut into blocks of size $u$ bits. A block is either a 0-block (full of zeros), or a 1-block (full of ones), or a non-monotonic block. $C_{0}$ may then be viewed as a sequence in any order of 0-runs (made of one or more successive 0-blocks), 1-runs (made of one or more 1-blocks), and non-monotonic runs (made of one or more non-monotonic blocks).

A 0 -run (1-run) is represented by one word of size $(u+2)$ bits where the two first bits are equar to 00 (11) and the last $u$ bits represent the run length in number of blocks. Non-monotonic runs are represented by as many words of size $(u+2)$ as there are blocks in the run, where the two first bits are equal to 01 , while the non-monotonic block ( $u$ bits) is copied into the last $u$ bits of the word. If the number of blocks in a 0 -run (1-run) is greater than $2^{u}$, then two or more successive words are necessary as in the RLC technique (see Section 3.1). Figure 8 illustrates the MCS technique on an example where $u=2$. 


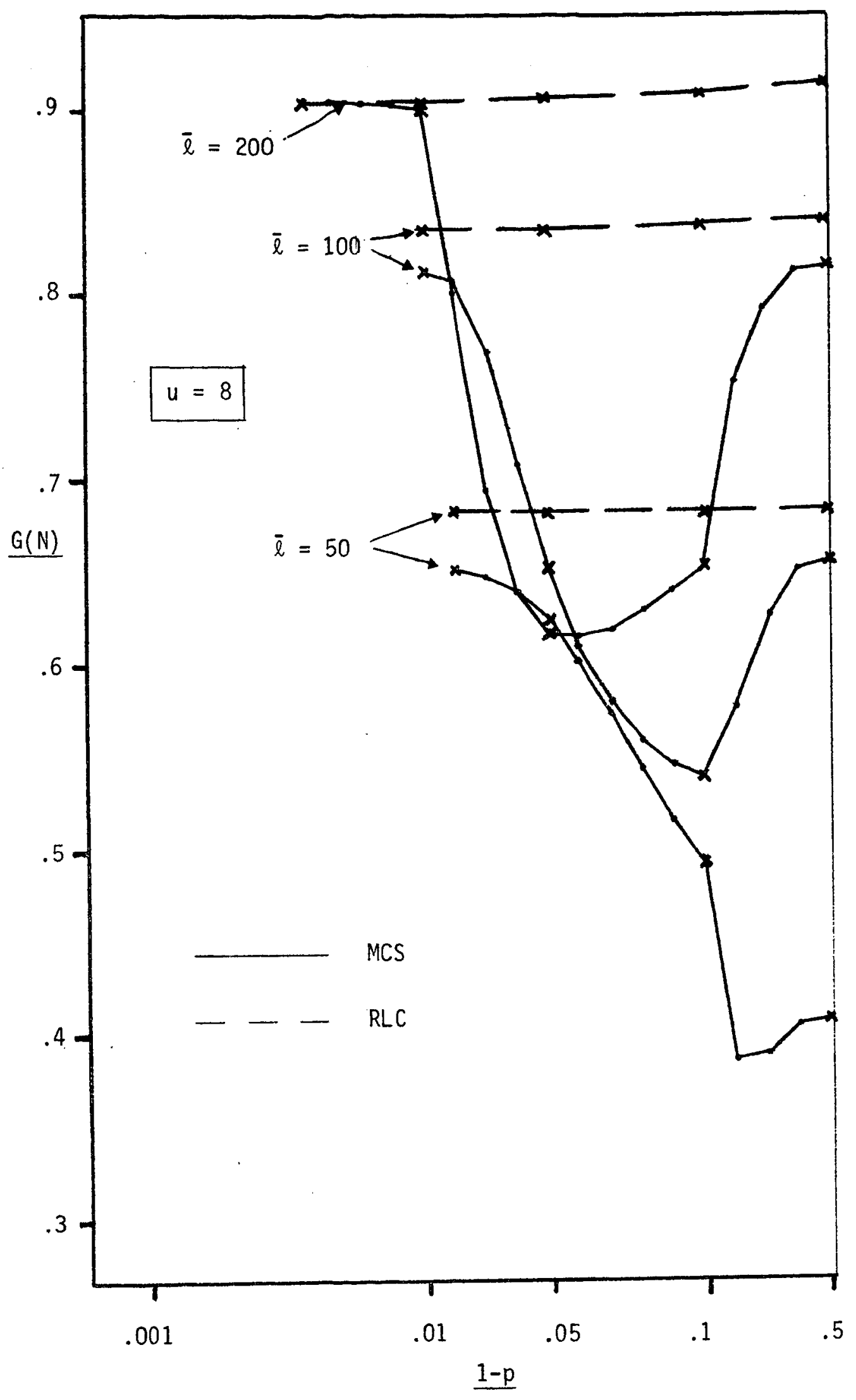

Figure 7 : RLC_and MCS : memory saving versus average

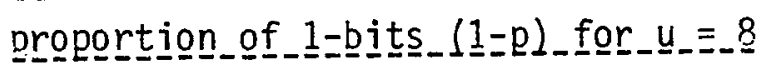




\section{\begin{tabular}{ll|l|l|l|l|l|l|l|l|l|l|l|l|l|l|l|l|}
\hline$c_{0}$ & 00 & 00 & 00 & 00 & 00 & 00 & 00 & 00 & 11 & 11 & 10 & 00 & 00 & 11 & 00 & 00 \\
\hline
\end{tabular}

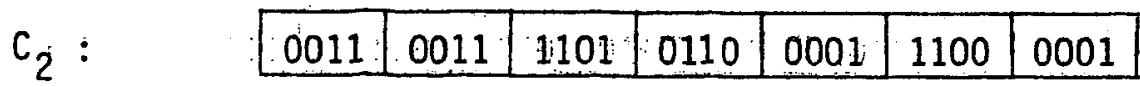

Figure_8 : MCS_technigue, 2 늨._. encoding.

Finally, note that this technique combines block compaction and run-length

\section{2 - Anàlysis}

If assumption A3 (see Section 3.2) is verified, then the sequence of blocks in string $C_{0}$ may be viewed as a Markov Chain with four states $\alpha, \beta, \gamma, \delta$ respectively corresponding to the occurrence in the string of a 0-block, a 1-block, a 10-block (non-monotonic block ending with a 0-bit) and a 01-block (non-monotonic block ending with a 1-bit).

The transition probabilities are given below as a function of $p_{0}, q_{1}$ (see Section 3.2) and of the following probabilities :

$a \triangleq P\left\{X_{i+u}=0 \mid X_{i}=0\right\}$ and $b \triangleq P\left\{X_{j+u}=1 \mid X_{i}=1\right\}$

a (b) is the probability a block ends with a 0-bit (1-bit) given the previlous block ended with a 0-bit (1-bit).

We have:

$$
\begin{aligned}
& P_{\alpha \alpha}=P_{\gamma \alpha}=p_{0}^{u} \\
& p_{\alpha \beta}=p_{\gamma \beta}=\left(1-p_{0}\right) q_{1}^{u} \\
& p_{\alpha \gamma}=p_{\gamma \gamma}=a-p_{0}^{u} \\
& P_{\alpha \delta}=P_{\gamma \delta}=1-a-\left(1-p_{0}\right) q_{1}^{u-1} \\
& P_{B \alpha}=P_{\delta \alpha}=\left(1-q_{1}\right) p_{0}^{u} \\
& P_{\beta \beta}=P_{\delta \beta}=q_{1}^{u} \\
& P_{B \gamma}=P_{\delta \gamma}=1-b-\left(1-q_{1}\right) p_{0}^{u-1} \\
& P_{B \delta}=P_{\delta \delta}=b-q_{1}^{u}
\end{aligned}
$$


One easily shows [5] that :

$$
\begin{aligned}
& a=\frac{\left(p_{0}+q_{1}-1\right)^{u}\left(1-p_{0}\right)+\left(1-q_{1}\right)}{2-p_{0}-q_{1}} \\
& b=\frac{\left(p_{0}+q_{1}-1\right)^{u}\left(1-q_{1}\right)+\left(1-p_{0}\right)}{2-p_{0}-q_{1}}
\end{aligned}
$$

Observe that, if $p_{0}=1-q_{1}=p$, we have, as expected, $a=1-b=p$ (the probability $x_{i}=0$ for all $i$, is equal to $p$; we are back to assumption $A 1$ of Section 2).

The resulting memory saving is given by (see Appendix $B$ ) :

$$
G(N)=1-\frac{u+2}{u}\left[\frac{p_{0}^{u}\left(1-p_{0}^{u}\right)}{1-p_{0}^{2^{u} u}}+\frac{q_{1}^{u}\left(1-q_{1}^{u}\right)}{1-q_{1}^{2^{u} u}}+\left(a-p_{0}^{u}\right)+\left(b-q_{1}^{u}\right)\right]
$$

\section{3 - Discussion}

Basically, as $u$ increases, $G(N)$ has the same behaviour with MCS as with RLC (see Figure 6) and with HCS (see Figure 3). Nevertheless $F_{2}(N)$ does not drop as much with MCS as with RLC, when $u$ increases. As for RLC, the larger the subchain length is, the larger $G(N)$ is (see Figure 7). Note however, $G(N)$ is very sensitive to the proportion $p$ of 0 -bits : a minimal value of the compaction ratio is observed for a value of $p$ increasing with the average subchain length ( $\bar{\ell}$, see Figure 7). Indeed, as $p$ decreases, (i) space is lost into the words for coding number of monotonic blocks which most of the time are much smaller than $2^{u}-1$, (ii) if $p$ is not too close to .5 , the saving due to the 1-bits compaction does not outweigh the coding overhead (equal to 2 bits).

\section{5 - EXPERIMENTAL VALIDATION OF THE ANALYSIS}

The "white noise" assumption of Section 2 (Assumption A1) is obviousiy pessimistic since it supposes no correlation exists between the values of two successive bits in the string. Assumption $A 3$ (see Section 3 ) is less restrictive and should lead to better predictions. In order to check how much pessimistic the theoretical performance is and to test whether the qualitative analysis conclusions are verified, the schemes performance has been estimated using data issued from a sample of 38 elementary maps actually stored in the geographic database described in [3]. This system has been designed as an aid 
for land-use planning of the Italian Emilia-Romagna region [2]. Each map is represented by a binary string of about $N=180,000$ bits which is compacted according to the three techniques.

For the Hierarchical Compaction Scheme $(H C O)$, the performance is computed by choosing for each map the optimal level of compaction $n_{0}$. Note that, in practice, one may prefer to choose the same level of compaction for all maps. Then the expected memory saving would be lower.

The "white noise" assumption implies the 0-runs (resp. 1-runs) are geometrically distributed with mean $1 /(1-p)$ (resp. 1/p). This assumption is far from being justified. Indeed, the average length of a 0 -run (taken over all maps) is found to be equal to 78.03 while a geometrical distribution would lead to a mean length equal to $22.73\langle p=.956$; for more details, see $[5])$.

The theoretical performance (from Eqs (2), (10) and (11)) and the actual performance (from measurement) are compared on Figure 9 where $G(N)$ is plotted versus $u$ for HCO, RLC and MCS.

From Figure 9 , one may draw the following conclusions :

(i) Quantitatively speaking, Assumption AI is very pessimistic : for HCS, the measured gain in memory is by far superior to that predicted by the analysis of Section 2. Assumption A3 allows a good performance prediction for RLC, but is still pessimistic as far as MCS is concerned. However, it allows a much better prediction of MCS performance than Assumption 1 does (see [4.7). Observe HCO and MCS provide a higher compaction ratio than RLC does.

(ii) Qualitatively speaking, an optimal block size exists for the three techniques and only RL.C performance is very sensitive to the block size. This was predicted by the analysis.

More results are given in [5]. In particular it is shown, for this sample of data, that $95 \%$ of the optimal memory saving is obtained if we choose the compaction level $n_{0}$ to be equal to 2 , with HCS. 


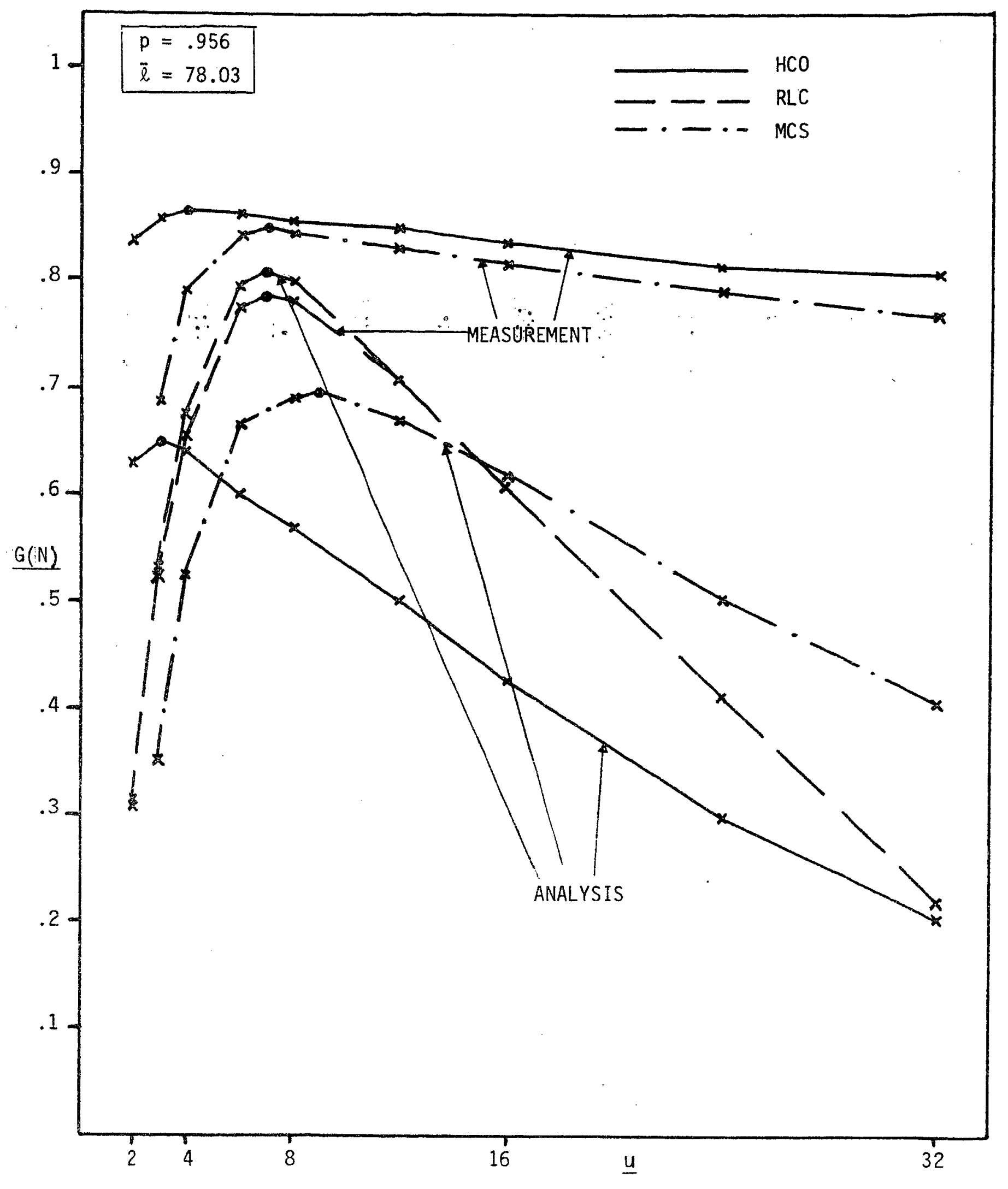

Figure 9 : HCO, RLC_. MCS : memory saving versus block size . Comparison between Ana]ys is and Measurement 


\section{6 - SIMULATION AND FURTHER COMPARATIVE STUDY}

The influence of the run lengths on the compaction ratio provided by the three techniques has been studied in [5] by means of a simulation experiment. Runs were generated according to an hyperexponential distribution. No correlation was assumed between any two successive runs. The performance predicted by this simulation is in agreement with that reported in Section 5 and obtained from an actual sample of data,sincel the error on the optimal memory saving is less than $7 \%[5]$.

Measurement showed the superiority of HCO and MCS in terms of memory saving, when the proportion of 0-bits is high ( $p=.956$, see Figure 9$)$. However, when $p$ decreases, RLC and MCS are expected to ouperform HCO, a variant of HCS in which only 0-bits are compacted. HC1, the other HCS variant in which both 0-bits and 1-bits are compacted should then provide a much better performance than $\mathrm{HCO}$ does, when $\mathrm{p}$ is as small as .5.

This is confirmed by the simulation study : in Figure $10, G(N)$ is plotted versus (I-p) as obtained from simulation for the four techniques, namely HCO, HC1, RLC and MCS. A block size of $u=8$ bits has been chosen for all schemes.

From Figure 10, clearly as $\mathrm{p}$ decreases beyond .9, HCO performance drops drastical1y, while HCI outperforms $H C O(G(N)$ stays larger than .7 , for $\bar{\ell}=100)$.

Observe, the simulation is in agreement with the analysis as far as RLC and MCS are concerned (see Figure 7). In particular, with RLC, the memory saving increases only slightly as $p$ decreases (when $p$ decreases, the probability it takes more than one block to code a 0-run is more and more negligible:: this event happens when a run has a length greater than 255 bits). In contrast to RLC, as predicted by the analysis, there is a minimal value of the memory saving (for $p \simeq .9$ if $\bar{\ell}=100$ ) with MCS.

Finally, note HC1 performance is extremely close to that of MCS and in particular, a minimum is observed for $G(N)$ for the same value of $p$ as for MCS. 


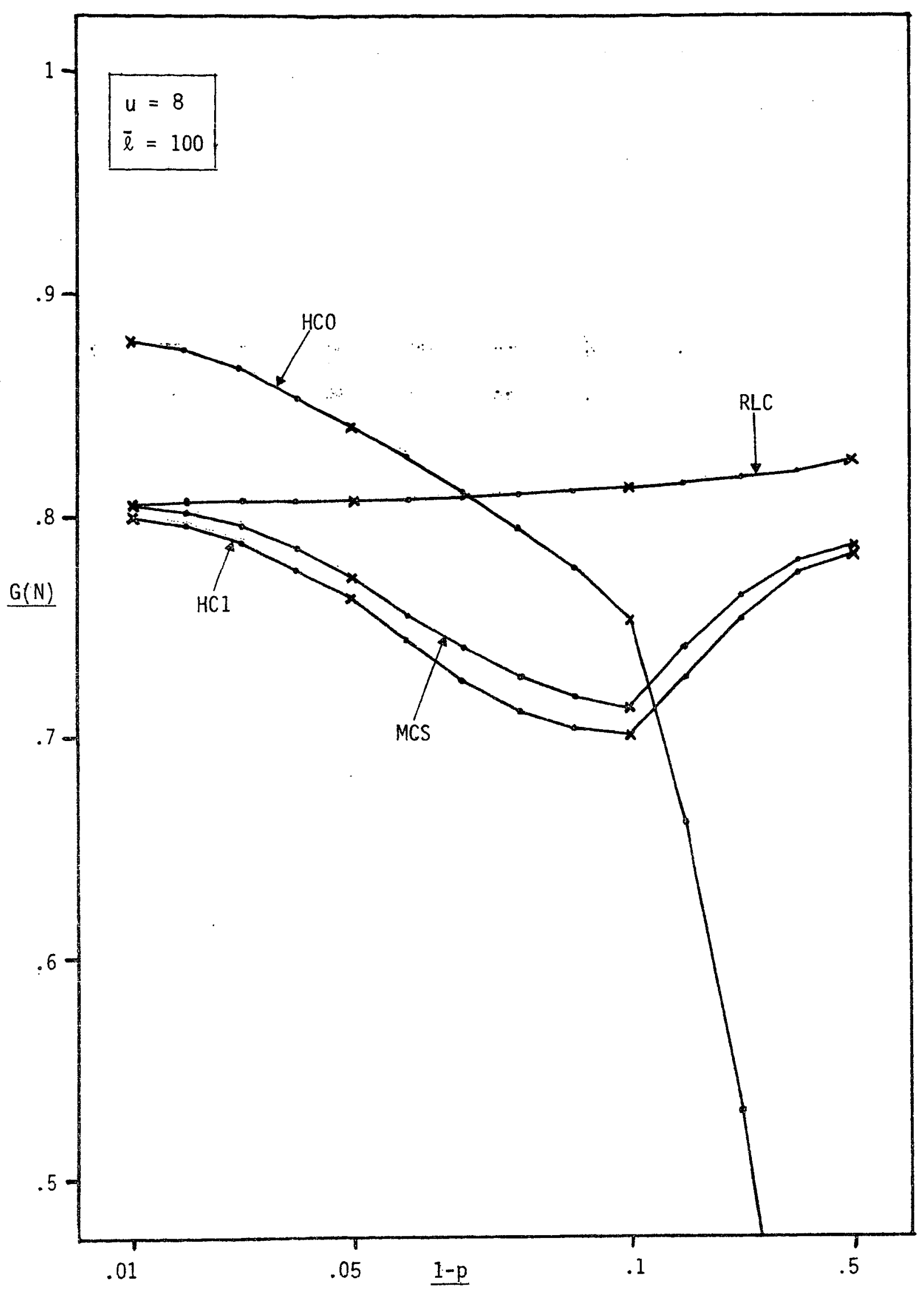

Figure $10: H C O, H C 1, R L C$ and MCS : memory saving versus average proportion of 1 -bits $(1-p)$ for $u$ _E 8 : SIMULATION_Result_s 


\section{7 - CONCLUSION}

In this paper, we have introduced a new compaction scheme called HCS, for cellular thematic maps decomposed into binary elementary maps. The memory saving obtained with this technique has been compared to that provided by two already existing schemes RLC [10] and MCS [3].

An analysis of the three techniques has been made, the validity of which was tested on a sample of actual data. The analysis turned out to give pessimistic predictions because of the restrictive assumptions on which it was based, especially for HCS.

A simulation experiment led to a more realistic prediction of the compaction ratio provided by these techniques, whatever the proportion of 0-bits in the maps is.

Compared to the other techniques, HCS presents the advantages of keeping set operations on maps such as intersection or union easy to be processed and of allowing variable scale (by varying the level of compaction within each map). 


\section{APPENDIX A : DERIVATION OF E(X) AND E(Y) FOR THE RLC TECHNIQUE} (proof of Eqs (7) and (8)).

* $E(X)=E\left(X_{0}\right)+E\left(X_{1}\right)$ where $E\left(X_{0}\right)$ and $E\left(X_{1}\right)$ respectively represent the average length of a 0 -run and a 1-run.

Let $P_{0, i}$ (resp. $P_{1, i}$ ) be the probability a 0 -run (resp. a 1-run) is of length $i$. Then, we have : :

$$
E(X)=\sum_{i=1}^{N} i\left(P_{0, i}+P_{1, i}\right)
$$

As an example, if assumption $A 3$ is verified, we have :

$$
P_{0, i}=\frac{p_{0}^{i-1}\left(1-p_{0}\right)}{1-p_{0}^{N}}
$$

Then, $E\left(X_{0}\right)=\sum_{i=1}^{N} i \frac{p_{0}^{i-1}\left(1-p_{0}\right)}{1-p_{0}^{N}}$, and when $N$ is large, it rapidly converges to $\frac{1}{1-p_{0}}$

Similarily, we have $E\left(x_{1}\right)=\sum_{i=1}^{N} i \frac{q_{1}^{i-1}\left(1-q_{1}\right)}{1-q_{1}^{N}}$ whose limit as $N$ goes to infinity is equal to $\frac{1}{1-q_{1}}$.

Then, as $N$ is large : $E(X) \simeq \frac{2-p_{0}-q_{1}}{\left(1-p_{0}\right)\left(1-q_{1}\right)}$

$$
\begin{aligned}
* E(Y) & =E\left(Y_{0}\right)+E\left(Y_{1}\right) \\
& \left.=u \sum_{j=1}^{j} j\left(Q_{0, j}+Q_{1, j}\right) \text { where } j_{\max }=\left\lceil\frac{N}{2^{u}-1}\right\rceil \text { and } Q_{0, j} \text { (resp. } Q_{1, j}\right) \text { is }
\end{aligned}
$$

the probability a 0 -run (resp. a 1-run) is included in the interval ]$\left.(j-1)\left(2^{u}-1\right), j\left(2^{u}-1\right)\right]$.

If: Assumption $A 3$ is verified, we have :

$$
Q_{0, j}=\left[p_{0}^{(j-1)\left(2^{u}-1\right)}-p_{0}^{j\left(2^{u}-1\right)}\right] /\left(1-p_{0}\right)^{N}
$$




$$
Q_{1, j}=\left[q_{1}^{(j-1)\left(2^{u}-1\right)}-q_{1}^{j\left(2^{u}-1\right)}\right] /\left(1-q_{1}\right)^{N}
$$

After some algebraic manipulations and assuming $N$ is Targe, we finally obtain :

$$
E(Y) \simeq u \sum_{j=0}^{i} \max ^{-1}\left[p_{0}^{j\left(2^{u}-1\right)}+q_{1}^{j\left(2^{u}-1\right)}\right]
$$


APPENDIX B : DERIVATION OF G(N) FOR THE MCS TECHNIQUE

(proof of Eq. (11))

Without loss of generality, assume string $C_{0}$ starts with a D-run. This happens with probability $\mathrm{p}_{\alpha}$.

Then, the average number of bits necessary to code $C_{0}$ (of length $N$ ) is:

$S_{\alpha}(N)=N\left[\frac{u+2}{u} \cdot \frac{E\left(Y_{\alpha}\right)}{E\left(X_{\alpha}\right)}\right] \cdots$

where $E\left(Y_{\alpha}\right)$ is the average number of words ( $u+2$ bits) necessary to code the subchain (of average length $E\left(X_{\alpha}\right)$ blocks of $u$ bits) located between the occurrence of the first 0-bitt of two successive 0-runs. We define similarily $E\left(X_{i}\right), E\left(Y_{i}\right), p_{i}$ and $S_{i}(N)$ for $i=\beta, \gamma, \delta$.

Then, we have :

$\frac{S(N)}{N}=\sum_{i=\alpha, \beta, Y, \delta} p_{i} \frac{S_{i}(N)}{N}=\frac{u+2}{u} \sum_{i=\alpha, \beta, \gamma, \delta} p_{i} \frac{E\left(Y_{i}\right)}{E\left(X_{i}\right)}$

Let us first derive the $E\left(Y_{i}\right)$ 's :

$E\left(Y_{\alpha}\right)=y_{\alpha}+\left[E\left(\alpha N_{\beta}\right) y_{\beta}+E\left(\alpha N_{\gamma}\right) y_{\gamma}+E\left(\alpha N_{\delta}\right) y_{\delta}\right]$

where $y_{i}$ is the average number of words (u+2 bits) necessary to code a $i$-run, and $E\left(i N_{j}\right)$ the average number of "visits" to state $j$ before coming back to state $i$.

We have [14]:

$E\left(i N_{j}\right)=\frac{E\left(X_{j}\right)}{E\left(X_{j}\right)}$

Then, $E\left(Y_{\alpha}\right)=E\left(X_{\alpha}\right) \sum_{i=\alpha, \beta, \gamma, \delta} \frac{y_{i}}{E\left(X_{i}\right)}$

From Eq. (B4), we obviously have:

$\frac{E\left(Y_{j}\right)}{E\left(X_{j}\right)}=\sum_{i=\alpha, \beta, \gamma, \delta} \frac{y_{i}}{E\left(X_{i}\right)}$, for all $j(j=\alpha, \beta, \gamma, \delta)$ 
we have :

Plugging Eq. (B5) into Eq. (B1) and observing that $\sum_{i=\alpha, \beta, \gamma, \delta} p_{i}=1$,

$$
\frac{S(N)}{N}=\frac{u+2}{u} \sum_{i=\alpha, \beta, \gamma, \delta} \frac{y_{i}}{E\left(X_{i}\right)}
$$

Let us now calculate the $E\left(X_{j}\right)$ 's $; E\left(X_{j}\right)$ is the average number of blocks (of length $u$ bits) in a $i$-subchain (starting with a $i$-run and ending with the last bit before the next $i-r u n)$. Then we have :

$$
E\left(x_{i}\right)=x_{i}+\sum_{j \neq i} x_{j} E\left(i N_{j}\right)
$$

where $x_{i}$ is the average number of blocks of a $i$-run.

Substituting Eq. (B3) into Eq. (B7) we finally have, as $N$ is large:

$$
\begin{aligned}
& E\left(X_{\alpha}\right) \simeq 1 /\left[p_{0}^{u}\left(1-p_{\delta}^{u}\right)\right] \\
& E\left(X_{\beta}\right) \simeq 1 /\left[q_{1}^{u}\left(1-q_{1}^{u}\right)\right] \\
& E\left(X_{\gamma}\right) \simeq 1 /\left[\left(a-p_{0}^{u}\right)\left(1-a+p_{0}^{u}\right)\right] \\
& E\left(X_{\delta}\right) \simeq 1 /\left[\left(b-q_{1}^{u}\right)\left(1-b+q_{1}^{u}\right)\right]
\end{aligned}
$$

Our remaining task is the calculation of the $y_{i}$ 's :

First, note that $y_{\gamma}=x_{\gamma}$ and $y_{\hat{o}}=x_{\delta}$, since to one non-monotonic block of string $C_{0}$, corresponds exactly one word in the compacted string. Since a 01 -run has length $k$ (blocks) with probability $\left(a-p_{0}^{u}\right)^{k-1}\left(1-a+p_{0}^{u}\right)$, as $N$ is large; then we have:

$$
y_{\gamma} \simeq 1 /\left(1-a+p_{0}^{u}\right)
$$

and similarily $y_{\delta} \simeq 1 /\left(1-b+q_{1}^{u}\right)$

One easily shows, as for the RLC technique (see Appendix 1), that

$$
\begin{aligned}
& y_{\alpha}=\sum_{j=0}^{j}\left(p_{0}^{u}\right)^{j 2^{u}} \quad \text { where } j_{\max }=\left[N / 2^{u}-1\right\rceil \\
& y_{\alpha} \simeq 1 /\left(1-p_{0}^{u 2^{u}}\right) \quad \text { as } N \text { is large }
\end{aligned}
$$

similarily, we have $y_{B} \simeq 1 /\left(1-q_{1}^{u 2^{u}}\right)$

Substituting Eqs (B8) to (B12) into Eq. (B5), we finally obtain Eq. (11). 


\section{REFERENCES}

[1] AMIDON E.L., AKIN G.S. - "Algorithmic selection of the best method for compressing map data strings" - Comm. ACM, Vo1. 14, n¹2, Dec. 1971, pp. 769-774.

[2] BONFATTI F., TIBERIO P., AMBROSINI L. - "A computer-based methodology for regional intervention planning" - IEEE - SMC Int. Conf. on Cybernetics and Society, Washington, Nov. 1976.

[3] BONFATTI F., TIBERIO P. - "Data management for thematic map generation" Computer and Graphics, Vol. 3, 1978, pp. 71-78.

[4] BOURSIER P., SCHOLL M. - "A new compaction scheme for map representation" Int. Conf. on Image Analysis and Processing, Pavia (Italy), 22-24 0ct. 1980.

[5] BOURSIER P. - "Représentation compacte en mémoire des cartes géographiques" Thèse de 3ème cycle, Université de Paris VI, (in preparation).

[6] BURTON W. - "Representation of many-sided polygons and polygonal lines for rapid processing"- Comm. ACM, Vol. 20, n³, March 1977, pp. 166-171.

[7] BYROM S.T., HARDGRAVE W.T. - "Representation of sets on mass storage devices for information retrieval systems" - National Computer Conference, 1973, pp. 245-250.

[8] COWAN D.D., GRAHAM J.W., WELCH J.W. - "The efficient representation of inverted 1ists" - Proc. 7th S.E. Conf. on Combinatorics, Graph Theory and Computing, 1976, pp. 257-272.

[9] FREEMAN H. - "Computer processing of line-drawing images" - Computing Surveys, Vo1.6, $n^{\circ} 1$, March 1974, pp. 57-97.

[10] KORTMANN C.M. - "Redundancy reduction : a practical method of data compression" - Proc. IEEE 55, 1967, pp. 253-263. 
[11] MERRILL R.D. - "Representation of contours and regions for efficient computer search" - Comm. ACM, Vol, 16, n², Feb. 1973, pp. 69-82.

[12] NAGY G., WAGLE S. - "Geographic data processing" - Computing Surveys, Vo1. 11, n², June 1979, pp. 139-181.

[13] NAGY G., WAGLE S. - "Approximation of polygonal maps by cellular maps" Comm. ACM, Vol. 22, n9, Sept. 1979, pp. 518-525.

[14] ROSS S.M. - "Applied probability models with optimization applications" Holden-Day, San Fransisco, 1970.

ACKNOWLEDGEMENTS : The authors thank Prof. F. BONFATTI from the University of Bologna (Italy) for having provided the data used in Section 5. They are also indebted to $\operatorname{Dr} D$. POTIER and P. LEBLANC from INRIA (France) for their constructive comments and to Dr F. BANCILHON from INRIA (France) for suggesting this problem. 
